\title{
The relationship of anxiety and stress with working men at private sector during covid-19 situation
}

Tuli Bhattacharya

Department of Education, Serampore Girls' College, Serampore, Hooghly, Pin: 712201, West Bengal, India (Affiliated to Calcutta University)

\begin{abstract}
The COVID-19 pandemic altogether affects public psychological wellness. Hence, observing and oversight of the populace emotional well-being during emergencies, for example, a pandemic is a prompt need. The point of this review is to break down the current research works and discoveries corresponding to the stress, tension and depression in everybody during the COVID-19 pandemic. Depression is a crippling ailment which builds the danger of self destruction. The subsequent mean levels found for pressure, tension, and depression was above levels considered non-obsessive. Ladies showed more significant levels of dread of COVID-19 than men. The measurable forecast for depression showed a solid match. This depression could be connected: both straightforwardly and emphatically by dread of COVID-19 and stress, and in a roundabout way, because of these two elements, decidedly intervened by tension. Our review finishes up by featuring the significant job that the complicated connections between dread, stress, and uneasiness can play in the improvement of depression indications and how they can be considered in programs pointed toward forestalling and reducing this problem. We propose some broad measures for diminishing apprehension of COVID-19 and push and recommend that particular projects be intended to control and conquer nervousness among students.
\end{abstract}

Keywords: Covid-19, anxiety, Stress, Working men

\author{
Article Publication \\ Published Online: 12-Nov-2021 \\ *Author's Correspondence \\ 8 Tuli Bhattacharya \\ 8 Department of Education, \\ Serampore Girls' College, \\ Serampore, Hooghly, West Bengal, \\ India (Affiliated to Calcutta \\ University) \\ $\bowtie$ tulibhattacharay.edu@gmail.com
}

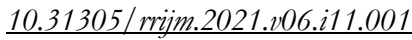

C 2021The Authors. Published by RESEARCH REVIEW International Journal of Multidisciplinary. This is an open access article under the CC BY-

NC-ND license

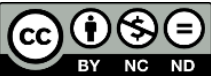

(https://creativecommons.org/licenses/ by-nc-nd/4.0/)

\section{Introduction}

Since December 2019, the flare-up of the coronavirus disease has been spreading quickly from China to different regions of the planet prompting intense irresistible pneumonia. In March 2020, the World Health Organization (WHO) reported the Covid disease, named COVID-19 disease, as a worldwide pandemic, which was brought about by the irresistible virus 'extreme intense respiratory disorder (SARS) coronavirus 2'. Like the past 2002 and 2012 viral flare-ups, SARS and Middle East Respiratory Syndrome (MERS-CoV), COVID-19 side effects included breathing hardships went with fever and hacking. Albeit extreme in the side effects showed, no settled antiviral treatment or immunization has been endorsed for the administration of COVID-19 to this date. On July 29, 2020, the Jordan Ministry of Health announced that the COVID-19 episode has brought about 1,187 affirmed cases in Jordan, and 11 instances of affirmed passing.

Social and physical removing, notwithstanding self-isolating, have been forced by numerous administrations around the world, because of the spread of the virus answered to be fundamentally through direct contact, for example drops spread by hacking or wheezing from a tainted individual. Quarantine is a viable measure in diminishing the spread of the virus around the world; nonetheless, it accompanies various considerable financial, social, and mental impacts. In result to this, few difficulties and concerns, including mental tensions, have been sanctioned on people. 
Mental health of people is a significant health concern, expected to be upset during pandemics, including the COVID-19 pandemic. As indicated by past research reports, during comparable viral episodes, a huge expansion in the danger of mental health issues among people occur, including uneasiness, misery and horrible pressure. During the new COVID-19 pandemic, expanded degrees of stress, outrage, uneasiness and wretchedness have been accounted for among people in various regions of the planet.

With an end goal to end the spread of the virus, most governments went to a few wellbeing lengths fluctuating from brief defer of exercises and occasions in instructive establishments to a total conclusion of schools and colleges. Distance learning turned into the course of training carried out in many nations, and obviously, encasing new concerns and difficulties for understudies. Subsequently, as a result to this significant change from the standard, mental health of undergrads was relied upon to be influenced.

The 2019 coronavirus disease (COVID-19) pandemic has caused fear, nervousness, stress and depression in people. in This Issue Students from countries hit hard by COVID-19 are often completely helpless as they face harsh isolation measures and have less room to adapt. The focus of this review was an analysis of the level of fear, stress, anxiety from COVID-19. and depression during the isolation of Ecuadorian students. and test possible indicators of depression using models drawn from our Logical Writing Review. A total of 640 students (72\%) aged 18 to 47 years $(M=21.69$; $S . D=4.093)$. The SARS-CoV-2 virus has a special effect on the respiratory system and is completely irresistible. with a long incubation period, the World Health Organization (WHO) has named coronavirus disease 2019 (COVID-19; Wu et al., 2020). It was first discovered in Wuhan, China in 2019, but its unique ability lies in its rapid distribution and development around the world. This world has led the WHO to view it as an epidemic (Rothan and Byrareddy, 2020). Recently, the microbe has become one of the most serious medical, social and financial problems in the world. Recently (Nicola et al., 2020) several studies have shown the impact that COVID-19 can have and its impact on human well-being. Due to the ability to provide a full range of emergency mental health services Especially in countries with large numbers of people affected by the disease (Fiorillo and Gorwood 2020). He has already begun to effectively study mental health issues such as poverty among people affected by COVID-19 (e.g. Duan and Zhu, 2020; Gao et al., 2020; Huang and Zhao, 2020). Problems that consist of side effects associated with a suppressed mind - despair, pity, self-deprecation. and futility - decreased confidence and lack of interest in everyday life. The problem is closely related to the lesser likelihood of achieving greater life goals for those who experience its detrimental effects. by destroying health and attempting to self-destruct (Antúnez and Vinet, 2012; Roh et al., 2020; Siegrist and Wege, 2020; Zhuo et al., 2020). and in the post-COVID-19 world We need to test the components associated with this problem.

\section{Methodology}

The psychological consequences of the epidemic are well understood as pre-epidemic psychological and psychological problems and the pathological consequences of the epidemic for these problems. Psychological responses to socialization and isolation response analysis public perceptions of people with COVID-19 infection and similar symptoms of infection outcomes. We searched for articles on coronavirus or COVID-19 and employment, COVID-19 and employee mental health, COVID-19 and mental health terms, Google Scholar, Web of Science and Semantic Scholar. Articles related to COVID-19 and depression techniques and COVID-19 in the workplace have been selected based on the relevance of our research topics. We searched articles that provided information on the effects of COVID-19 on employees' mental health. We highlight articles published between December 2019 and March 2020, not biomedical articles. Our goal is to analyze articles that help build bridges between epidemiology, psychology, and human resource management. Due to lack of information about the effects of COVID-19 on employee mental health. Therefore, we must investigate recent outbreaks such as SARS (Severe Acute Respiratory Syndrome) and Ebola. In this case, we searched articles that linked the epidemic to mental health All peer-reviewed articles are included in this article and are listed in the bibliography. 


\section{Fundamental concepts}

The resulting mental health and mental well-being is ranked 10th most frequently surveyed during the COVID-19 outbreak. From nervousness, suffering, stress after a terrible incident. and moderately high stress levels for all people and healthcare professionals during the global COVID-19 pandemic. In Asia, several Central Paying Countries (MICs) are facing enormous financial constraints and limited clinical assets. to keep up with the physical and mental boom during the pandemic, it also spread to North America. a sudden change in financial stability during COVID19 An increase in the rate of self-destruction is expected. During the pandemic, the Asia-Pacific Disaster Mental Health Network began developing a mental health plan for direct survey in Asia to examine the mental health of Asians living in middle-income countries in order to help limit the capacity of various health systems. In response to COVID-19, past research has mainly focused on mental health issues in selected Asian countries during the pandemic without review.

When no comparable reviews of the physical and mental health of Asians living in middle-income countries (COVID-19) were found during the COVID-19 outbreak, this review aims to examine the physical and mental health impacts of the pandemic in 7 middle-income countries. (China. Iran, Malaysia, Pakistan, Philippines, Thailand and Vietnam). Understand the attention and preventive measures against COVID-19, as well as the perception of protective factors and hazards associated with mental health consequences.

\section{Dread of COVID-19, Stress, Anxiety, and Depression}

Epidemiological agencies have had to pass strict laws to prevent the spread of many (Adhikari et al., 2020). In terms of the number of infections, in terms of infection and death rates, for example, China, Italy, Spain and Ecuador have announced large-scale self-quarantine and more closures. What kind of residents need to stay at home has a real impact on the daily environment of their population. And it gets in the way, especially in less affluent countries. In Latin America, for example, some diseases, such as the spread of fragility, the development of infected patients or drug resistance, or the lack of antibodies to fight the disease, cause fear among the population (Orellana, Orellana, 2020; Ornell et al., 2020; Rodríguez-Rey et al. al., 2020). The compromise has been effective in disasters caused by past disasters such as SARS (Reynolds et al., 2008) or the Middle East coronavirus respiratory syndrome (MERS$\mathrm{CoV}$; Bukhari et al., 2016). Serious consequences around the world where the COVID-19 epidemic was created in terms of tolerance, well-being, prosperity and human development. Ahorsu et al. (2020) developed a scale for measuring this microbial fear based on the current logical writing: COVID-19 Fear Scale (FCV-19S). This scale is used in many countries, such as Iran (Aliami et al., 2020), Bangladesh (Shakib et al., 2020), Italy (Sorasi et al., 2020), Turkey (Satisi et al., 2020), Russia and Belarus (Regnik et al., 2020), Israel (Tzur Bitan et al., 2020), Peru (HuarcayaVictoria et al., 2020), and Paraguay (Barrios et al., 2020).

Most studies have also identified a link between COVID-19 (Mertens et al., 2020) and less significant depression due to panic and nervousness. Both the Hospital Anxiety Scale and the Depression Scale (HADS; Ahorsu et al., 2020; Alyami et al., 2020) and the latest Depression and Anxiety Stress Scale (DASS-21) were used. It is believed to be more related to tension and stress. And it is less important in depression (Tzur Bitan et al., 2020), although there is less link between fear and depression. Examples of self-destruction in society for fear of COVID-19 have been described (Mamun and Griffiths, 2020). Apart from this, the rate of epidemics and epidemics is increasing day by day due to the blockade of the people. Media communication can influence the progression of mental health problems (Duan and Zhu, 2020; Gao et al., 2020). Chinese researchers have found moderate to severe side effects of anxiety, stress and depression in the Chinese population (Huang and Zhao, 2020).

The link between stress and tension has been documented for quite some time through a depressing logical writing. The hypothetical model based on logical evidence is the association of social and environmental stress with the internal biological cycle that determines the pathogenesis of depression (e.g., Slavich and Irwin, 2014; Park et al., 2019). Longitudinal studies in children also suggest that blood pressure predicts depression (e.g., Auguston and Rudolph, 2011). We understand that there is a similar relationship between stress and depression, especially in 
unpleasant situations (Díaz et al., 2012). They often display undeniable levels of fear and anxiety (Forbes et al., 2012 al., 2010). Anxiety and depression are known to be linked. (Jansson-Fröjmark and Lindblom, 2008), and both powers point to each other (Jacobson, Newman, 2017; Hovenkamp-Hermelink et al., 2019).

The absence of occasions, end of the week exercises just as exercises with loved ones produce the most noteworthy way of life weight on members showing first world concerns, which might be identified with territorial conveyance and to the white - collar profile of members. The main remote working stressors was the detachment of work and everyday life in Europe and North America, though feeling disengaged was exceptionally positioned in Asia, South America, and different locales. Troubles in isolating work and family lives, feeling confined absence of security just as over working overwhelm $d$ the rundown of remote working stressors around the world. Strains on family connections involved the center positions of the stressor list in Europe and North America and the lower positions in any remaining areas. The female members scored the remote working stressors fundamentally higher than their male and various companions, demonstrating a higher burden on ladies while working distantly. Especially females from North America were the most focused on population. Remote working pressure showed solid sexual orientation disparity. Post-pandemic worries of members demonstrated more word related (scholastic versus nonscholarly), hierarchic (having administrative role, having the option to set own needs or not) and segment (age related) incongruities than sex inconsistencies.

Social tension has been the most pervasive worry among all members. Segment investigation showed that the portions of various post-pandemic concerns differ contingent upon the age of the members, as well. Members with administrative roles announced that they were happy with the exhibition of their representatives while working distantly. Most of all members communicated that they approach adequate apparatuses to achieve their work, while criticism from directors and associates just as other data were the most wanted however missing info. Data straightforwardness and preparing about pandemic dangers expanded nonparticipant for nonstop work in the non - scholarly area, however don't cure the worries of members.

\section{Reviews of literature}

According to Grant \& Booth's (2009) taxonomy, the method used in this article is a brief description of the literature. Which provides an overview of the latest and greatest literature? And covers a wide range of issues at different levels of maturity and reach This article shows the COVID-19 coronavirus around the world as we write this. We specifically study the effects on employee mental health. With stress we see that screening factors and companies can take steps to reduce the effects of COVID-19 on the mental health of employees. Descriptive style is a key aspect of this type of review (Grant \& Booth, 2009) which we have used in this article. Psychological distress consists of a series of psychological and behavioral symptoms that are distributed over a period of time (Marchand, 2004), where depression is a mental disorder. It is characterized by a persistent loss of mood and attention (Bonde, 2008), constant feelings of sadness, negative emotions, and difficulty performing daily tasks (Cummins 2015). Psychosis can lead to major depression (Marchund, 2004), while depression can lead to serious consequences such as suicide (Beck \& Alford, 2009; Cummins 2015).

Mental health disorders and depression are the result of intense or endless stress that cannot be addressed. This is mainly due to the fact that people find it difficult to cope with stressful life events (Cummins, 2015; Drapeau 2011; Marchand, 2004). The current epidemic is a serious source of stress for populations around the world.

The COVID-19 epidemic could be associated with a number of stressors that could threaten the mental health of workers. During and after this outbreak, in this section, we differentiate between stresses during coronavirus outbreaks. What can happen after this epidemic is not a problem that everyone is going through. This is an obvious consequence of the problem (Mirowsky \& Ross, 2003). Understanding the problem is important so that solutions can be found to help employees and organizations reduce the risk of mental health problems. This is the main purpose of this article. 
Analysis

\section{Adjusting to the changes}

This episode highlights how many people can better cope with the current situation by remaining silent or by finding ways to remain silent by avoiding frightening burdens or provocations on television or online media. Develop or discover old and obvious interests and distortions inherent in the phone or computer. A new and challenging task for many people who use it to understand how to work remotely. Another useful strategy is to find ways to calm your brain. The academic foundation for students is changing the way we prepare, connect and deliver computerbased assessments. The expected situation is very painful. As we approach epidemics, mental health professionals should also expect improvements in mental health. Mental disorders can be accompanied by stress during the recovery period, as well as commitment, compulsion, family stress, and so on. For families isolated from patients with COVID-19 complications, separation can be severely affected from a psychological perspective that publicly triggers anxiety, weakness, and depression. There will be situations when one has to face the unexpected death of a relative or be prepared for unintended consequences. As data on the effects of COVID-19 are rapidly being updated, there are concerns about the potential direct neurological consequences of COVID-19 infection, the relationship between mental prescription and the drugs used. Treat Infections In most patients, in conjunction with medication, changes in ECT delivery may be required, while other physical therapy strategies for highly demonstrated COVID19 patients are another area that needs to be clearly understood.

\section{Results}

For sure COVID-19 has affected the psychological well-being of the whole local area in some way. This has made a phenomenal emotional well-being challenge despite restricted expert assets. This likewise implies that we need to think past regular procedures. Cutting edge staff should be prepared in straightforward and arising proof based systems of appraisal and the board. As of now, the therapeutic measures recommended for COVID-19 have just exact proof. We need to make do with what is accessible, yet continually update ourselves, nearly day by day. All the while, we need to draw in with non-mental clinical experts to work with the mental administration of patients with COVID- 19 disease and co-dreary actual sicknesses. Computerized empowered learning and backing for emotional wellness issues should be considered for all non-mental clinical experts and bleeding edge work force. Web based learning for are similarly valuable for standard updates for specialists too. Regardless of the different interruptions in routine clinical practice, particularly the standard outpatient services, this emergency has hurled elective and inventive ways to deal with offer psychosocial support and nonstop care for patients with mental issues or mental pain. Online computerized correspondence stages have turned into a shelter for follow-up contact with patients just as to disperse preparing to experts working even in far off settings.

Managing Teleworking and Anticipating Social Exclusion to prevent mental health disorders of telemedicine workers during COVID-19, associations must develop legal processes to assist agents during hierarchical transitions. The investigation by Greer and Payne (2014) set out certain procedures recognized by telecommunications. This can help overcome the difficulty of working remotely. This method involves continuous communication with employees and managers. while working remotely based on assumptions, work progress and availability, Agents not only need to adapt to align their plans to complete tasks and requirements, but also provide excellent innovative equipment. Work on the job and communicate with like -minded directors and people. and reduce stress Telecommunications must also be prepared to innovate in the workplace and interact when away from the work environment. This reduces stress levels (Greer and Payne, 2014).

\section{Conclusion}

The absence of occasions, end of the week exercises just as exercises with loved ones create the most noteworthy way of life weight on members demonstrating first world concerns, which might be identified with provincial dispersion and to the white - collar profile of members. The main remote working stressors was the partition of work and everyday life in Europe and North America, though feeling confined was exceptionally positioned in Asia, South America, and different districts. Challenges in isolating work and family lives, feeling detached, absence of 
security just as over working overwhelm $\mathrm{d}$ the rundown of remote working stressors around the world. Strains on family connections involved the center positions of the stressor list in Europe and North America and the lower positions in any remaining districts. The female members scored the remote working stressors fundamentally higher than their male and different friends, demonstrating a higher burden on ladies while working distantly. Especially females from North America were the most focused on population. Remote working pressure showed solid sex disparity. Post-pandemic worries of members demonstrated more word related (scholastic versus non-scholarly), hierarchic (having administrative role, having the option to set own needs or not) and segment (age - related) aberrations than sexual orientation incongruities.

Social uneasiness has been the most common worry among all members. Segment examination showed that the portions of various post-pandemic concerns differ contingent upon the age of the members, as well. Members with administrative roles proclaimed that they were happy with the presentation of their representatives while working distantly. Most of all members communicated that they approach adequate devices to achieve their work, while criticism from supervisors and associates just as other data were the most wanted yet missing information. Data straightforwardness and preparing about pandemic dangers expanded nonparticipant for constant work in the non - scholastic area, however don't cure the worries of members.

\section{References}

Bai Y, Lin CC, Lin CY, et al. : Survey of stress reactions among health care workers involved with the SARS outbreak. Psycbiatr Serv. 2004;55(9):1055-1057. 10.1176/appi.ps.55.9.1055

Bao Y, Sun Y, Meng S, et al. : 2019-nCoV epidemic: address mental health care to empower society. Lancet. 2020;395(10224):e37-e38. 10.1016/S0140-6736(20)30309-3

Beck AT, Alford BA: Depression: Causes and treatment. University of Pennsylvania Press.2009;405 10.1080/15323269.2010.492268

Benson DW, Dix KS: Pandemic preparations for the workplace. Colorado Lanyer. 2009;38:49 Bitanihirwe BK: Monitoring and managing mental health in the wake of Ebola. Commentary. Ann Ist Super Sanita. 2016;52(3):320-322. 10.4415/ANN_16_03_02

Bonde JP: Psychosocial factors at work and risk of depression: a systematic review of the epidemiological evidence. Occup Environ Med. 2008;65(7):438-445. 10.1136/oem.2007.038430

Bontcheva K, Gorrell G, Wessels B: Social media and information overload: Survey results. arXiv preprint arXiv:1306.0813. 2013.

Braunack-Mayer A, Tooher R, Collins JE, et al. : Understanding the school community's response to school closures during the H1N1 2009 influenza pandemic. BMC public health. 2013;13(1):344 10.1186/1471-2458-13-344

Brooks SK, Dunn R, Amlôt R, et al. : A Systematic, Thematic Review of Social and Occupational Factors Associated With Psychological Outcomes in Healthcare Employees During an Infectious Disease Outbreak. J Occup Environ Med. 2018;60(3):248-257. 10.1097/JOM.0000000000001235

Brooks SK, Webster RK, Smith LE, et al. : The psychological impact of quarantine and how to reduce it: rapid review of the evidence. Lancet. 2020;395(10227):912-920. 10.1016/S0140-6736(20)30460-8

Brug J, Aro AR, Oenema A, et al. : SARS risk perception, knowledge, precautions, and information sources, the Netherlands. Emerg Infect Dis. 2004;10(8):1486-9. 10.3201/eid1008.040283

Chan M: Ebola virus disease in West Africa--no early end to the outbreak. N Engl J Med. 2014;371(13):1183-1185. 10.1056/NEJMp1409859

Chen CS, Wu HY, Yang P, et al. : Psychological distress of nurses in Taiwan who worked during the outbreak of SARS. Psychiatr Serv. 2005;56(1):76-9. 10.1176/appi.ps.56.1.76 
Chiu M, Amartey A, Wang X, et al. : Trends in objectively measured and perceived mental health and use of mental health services: a population-based study in Ontario, 2002-2014. CMAJ. 2020;192(13):E329-E337.

CNA: China says 6 health workers died from coronavirus, 1,716 infected [Online]. Asia: Channel News Asia.2020; [Accessed March 20, 2020]. Reference Source [Google Scholar]

Corkery M, Maheshwari S: Is There Really a Toilet Paper Shortage?New York: The New York Times.2020; [Accessed March 16, 2020]. Reference Source [Google Scholar] 